Check for updates

Cite this: RSC Adv., 2017, 7, 56016

Received 22nd October 2017

Accepted 5th December 2017

DOI: $10.1039 / c 7 r a 11650 k$

rsc.li/rsc-advances

\title{
Colorimetry and phase transition characteristics in sensing fluoride anion based on hydrazide organogelators $\uparrow$
}

\author{
Xia Ran, ${ }^{a}$ Qiongqiong Gao, ${ }^{a}$ Yu Zhang ${ }^{* b}$ and Lijun Guo (D) *a
}

\begin{abstract}
The fluoride anion sensing properties of BNB-t4 and BNBC-t8 consisting of hydrazide and azobenzene moieties both in solution and gel state, and the involved binding mechanism have been systematically investigated in this work. The remarkable changes in the absorption of receptor BNB-t4 with a terminal hydroxyl group demonstrate a colorimetric chemosensor with a higher sensitivity in sensing fluoride anions than that of BNBC-t8 with a terminal methoxy group. The detection limit of BNB-t4 for the analysis of $\mathrm{F}^{-}$can reach as low as $4.27 \times 10^{-8} \mathrm{M}$, while this value is $2.02 \times 10^{-6} \mathrm{M}$ for BNBC-t8. The results indicate that the $\mathrm{F}^{-}$ion interacts with the amidic $-\mathrm{NH}$ and hydroxyl proton of $\mathrm{BNB}-\mathrm{t} 4$ via hydrogen-bonding to give the stable $1: 2$ complex at the first equilibrium state, and further addition of $\mathrm{F}^{-}$can induce deprotonation by forming $\mathrm{HF}_{2}{ }^{-}$to establish a second equilibrium state. Meanwhile, the gel-sol transition of BNB-t4 has been successfully applied in sensing fluoride anions and thus makes BNB-t4 a naked-eye sensor. The color change of BNB-t4 induced by binding fluoride anions can be safely switched off with the addition of $\mathrm{HSO}_{4}{ }^{-}$, demonstrating an OFF-ON-OFF colorimetric sensor with a good reversibility.
\end{abstract}

\section{Introduction}

Anions play essential roles in many environmental, biological and industrial systems, and anion sensing has become one of the most attractive fields of supramolecular chemistry. ${ }^{1-4}$ Fluoride is a small inorganic anion often added to toothpaste due to its beneficial role in dental health, and has also been used in the treatment of osteoporosis. ${ }^{5,6}$ The right amount of fluoride can prevent dental cavities, and in excess can lead to skeletal and dental fluorosis in humans. ${ }^{7}$ Hence, regulation and detection of fluoride are crucial in various environmental and health applications. In past decades, the field of anion sensors based on different signaling mechanisms, such as intramolecular charge transfer, ${ }^{8-10}$ twisted intramolecular charge transfer, ${ }^{11}$ photoinduced electron transfer, ${ }^{12-16}$ metal-ligand charge transfer, ${ }^{17-20}$ fluorescence resonance energy transfer, ${ }^{21-25}$ excited state proton transfer, ${ }^{26-31}$ and excimer/exciplex formation, ${ }^{32,33}$ has been well established and studied. However, most of the reported probes suffer from low sensitivity, slow response, or complicated synthetic procedures. Therefore, it is still a challenge to develop a highly selective and rapid detection method.

${ }^{a}$ Institute of Micro/Nano Photonic Materials and Applications, Henan University, Kaifeng 475004, People's Republic of China. E-mail: juneguo@henu.edu.cn ${ }^{b}$ Institute of Plant Stress Biology, Henan University, Kaifeng 475004, People's Republic of China.E-mail: qikeli@hotmail.com

$\dagger$ Electronic supplementary information (ESI) available. See DOI: 10.1039/c7ra11650k
To date, many synthetic chemical receptors incorporating neutral or cationic $\mathrm{N}-\mathrm{H} / \mathrm{F}^{-}$hydrogen bonding donor groups (e.g., pyrrole, indole, ammonium, guanidinium, urea, thiourea, and hydrazide) have been reported..$^{\mathbf{2 0 , 3 4 - 3 8}}$ In contrast, receptors based on $\mathrm{O}-\mathrm{H} / \mathrm{F}^{-}$hydrogen bond interactions have been less exploited, ${ }^{39-42}$ even though $\mathrm{O}-\mathrm{H}$ shows higher acidity than $\mathrm{N}-\mathrm{H}$ and the $\mathrm{O}-\mathrm{H} /$ anion hydrogen bonding is almost as crucial as the ubiquitous $\mathrm{N}-\mathrm{H}$ /anion interaction. ${ }^{43}$

Stimuli-responsive organogel, structurally controlled by the assemblies of low-molecular-weight gelators (LMWGs) through noncovalent interactions is one of the most attractive examples due to its unique properties. ${ }^{44}$ The gelation behavior involves the molecule self-assembly into three dimensional structures and is usually influenced by the external conditions, and thus can be tuned by physical and chemical stimuli, such as temperature, ${ }^{45} \mathrm{UV} /$ visible light, ${ }^{46}$ ions, ${ }^{47-56}$ ultrasound, ${ }^{57}$ and so on. Therefore, the self-assembly behavior, sol-gel transition, and colorimetric/fluorescent change of LMWGs in gel or solution can be employed as molecular sensors in monitoring external stimuli. To create simple, convenient, and economical sensors requires successful fabrication of small molecular anion sensors into colorimetric test kits. ${ }^{44}$ So far, a few reports on anion-tuning organogels have been reported for detecting fluoride ion. ${ }^{47-49}$ For instance, Lee and co-workers found that translucent colorless gel can be changed to liquid and showed a strong greenish fluorescence with the presence of fluoride anion. ${ }^{47} \mathrm{Wu}$ and co-workers developed a specific colorimetric and fluorimetric sensor for detecting fluoride anion. ${ }^{48} \mathrm{Jiang}$ and 
<smiles>COc1cc(C(=O)NNC(=O)c2ccc(/N=N/c3ccc(O)cc3)cc2)cc(OC)c1OC</smiles>

BNB-t4<smiles>CCOc1cc(C(=O)NNC(=O)c2ccc(/N=N/c3ccc(OC)cc3)cc2)cc(OCC)c1OCCF</smiles>

BNBC-t8

Scheme 1 Molecular structures of BNB-t4 and BNBC-t8.

co-workers demonstrated that organogels based on salicylidene Schiff base showed a highly selective dual-responsive behavior to $\mathrm{Zn}^{2+}$ and $\mathrm{F}^{-}$, respectively. ${ }^{49}$ In the meantime, the detection limit for fluoride anion in sol phase has been improved to micromole level, which is low enough to be applied in environmental or biological fields. ${ }^{2}$ Although previous works presented a wide variety of anion sensors based on electrostatic interactions, hydrogen bond donor groups, Lewis acid groups and hydrophobic interactions, it is still a challenge to design and synthesize anion organogelators with specific selectivity and high sensitivity for sensing certain anions, because of their lower charge to radius ratio and highly solvated nature. ${ }^{44}$ Especially, the gelators showing both reversible and highly sensitive colorimetric changes and sol-gel transition by anion stimuli are still limited to date.

Here, as an attempt to obtain a smart fluoride anionresponsive gelator with potential anion sensing applications, we had designed and synthesized a gelator bearing phenol $\mathrm{O}-\mathrm{H}$ and hydrazide for hydrogen bond donor subgroups, $N$ - $(3,4,5-$ tributoxyphenyl)- $N^{\prime}-4$-[(4-hydroxyphenyl)azophenyl] benzohydrazide (BNB-t4), and a control molecule, $N$-(3,4,5-octanoxyphenyl)- $N^{\prime}-4$-[(4-methoxyphenyl)azophenyl] benzohydrazide (BNBC-t8) (Scheme 1). ${ }^{46,58}$ The characteristics and binding mechanism of BNB-t4 with fluoride anion have been systematically investigated in this work. The results indicate that BNB-t4 gels exhibit both colorimetric and gel-sol transition sensing properties upon addition of fluoride anion. The remarkable change in the absorption of receptor BNB-t4 with terminal hydroxyl groups demonstrates a colorimetric chemosensor with higher sensitivity in sensing fluoride anion than that of BNBCt8 with terminal methoxy groups. In addition, the color change of BNB-t4 induced by binding fluoride anion can also be safely switched off with the addition of $\mathrm{HSO}_{4}{ }^{-}$, demonstrating an excellent $\mathrm{OFF}-\mathrm{ON}-\mathrm{OFF}$ colorimetric sensor with a robust reversibility.

\section{Results and discussion}

During anion recognition, the interaction between receptor and target anion can lead to changes in molecular electronic ground state, and thus in the absorption properties. To this end, UV-vis spectrometry was performed to evaluate the recognition ability of BNB-t4 toward $\mathrm{F}^{-}, \mathrm{Cl}^{-}, \mathrm{Br}^{-}, \mathrm{I}^{-}, \mathrm{CH}_{3} \mathrm{COO}^{-}\left(\mathrm{AcO}^{-}\right), \mathrm{HSO}_{4}{ }^{-}$and $\mathrm{H}_{2} \mathrm{PO}_{4}{ }^{-}$in $\mathrm{CHCl}_{3}$ and DMSO, respectively (using their tetrabutylammonium salts as the sources). As shown in Fig. 1a, the selective binding of BNB-t4 in $\mathrm{CHCl}_{3}$ with anions displayed intense variations in absorption spectra upon addition of the same amount of $\mathrm{F}^{-}, \mathrm{AcO}^{-}$and $\mathrm{H}_{2} \mathrm{PO}_{4}{ }^{-}$at room temperature, while no obvious colorimetric changes were observed in the presence of $\mathrm{Cl}^{-}, \mathrm{Br}^{-}, \mathrm{I}^{-}$and $\mathrm{HSO}_{4}{ }^{-}$. With the addition of 30 equiv. $\mathrm{F}^{-}$ion, the characteristic absorption maximum at $356 \mathrm{~nm}$ of BNB-t4 is dramatically decreased and a new broad band around $460 \mathrm{~nm}$ appears, indicating the formation of new complexes. The color of solution is also changed from light yellow to orange, which could be easily observed by the naked eyes. The selective binding of BNB-t4 in DMSO with anions shows the similar phenomena, in addition to having a deeper color change (from light yellow to crimson upon addition of 30 equiv. of $\mathrm{F}^{-}, \mathrm{AcO}^{-}, \mathrm{H}_{2} \mathrm{PO}_{4}^{-}$and $\mathrm{OH}^{-}$) (Fig. S1 $\dagger$ ). Furthermore, the $\mathrm{pH}$ dependence of BNB-t4 in HEPES buffer solution system was examined using UV-vis spectroscopy. The results indicate that BNB-t4 binding process with $\mathrm{F}^{-}$can take place at the $\mathrm{pH}$ value ranging from 11 to 14 (Fig. S2 $\dagger$ ). However, when $\mathrm{pH}$ value of buffer solutions was 11, the characteristic absorption
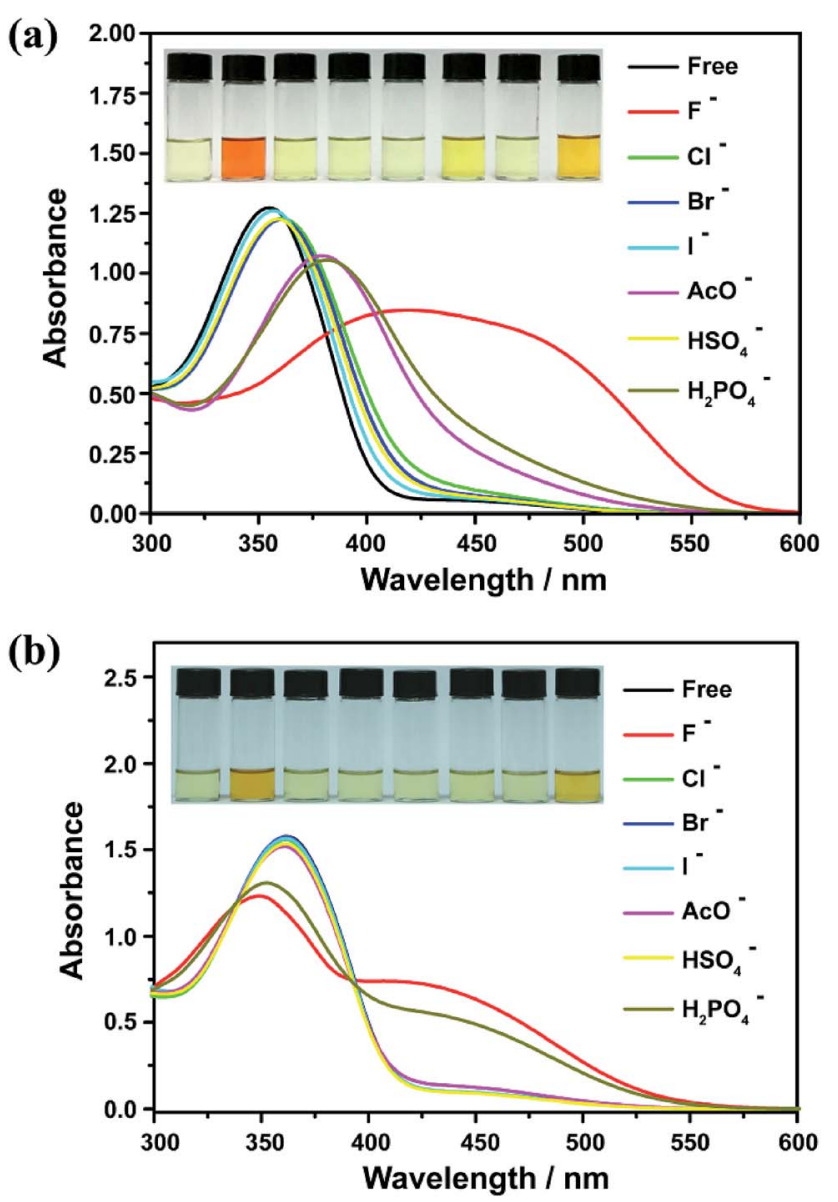

Fig. 1 UV-vis spectra of (a) BNB-t4 $\left(1 \times 10^{-4} \mathrm{~mol} \mathrm{~L}^{-1}\right)$ and (b) BNBC-t8 $\left(1 \times 10^{-4} \mathrm{~mol} \mathrm{~L}^{-1}\right)$ in $\mathrm{CHCl}_{3}$ upon addition of 30 equiv. of various anions $\left(\mathrm{F}^{-}, \mathrm{Cl}^{-}, \mathrm{Br}^{-}, \mathrm{I}^{-}, \mathrm{AcO}^{-}, \mathrm{HSO}_{4}^{-}\right.$and $\left.\mathrm{H}_{2} \mathrm{PO}_{4}^{-}\right)$. Inset: the corresponding images of BNB-t4 and BNBC-t8 $\left(1 \times 10^{-4} \mathrm{~mol} \mathrm{~L}^{-1}\right)$ in $\mathrm{CHCl}_{3}$ upon addition of 30 equiv. of various anions (from left to right: free, $\mathrm{F}^{-}$, $\mathrm{Cl}^{-}, \mathrm{Br}^{-}, \mathrm{I}^{-}, \mathrm{AcO}^{-}, \mathrm{HSO}_{4}^{-}$and $\mathrm{H}_{2} \mathrm{PO}_{4}^{-}$). 
maximum at $485 \mathrm{~nm}$ of BNB-t4 in HEPES buffer solution system was almost unchanged with the addition of $\mathrm{OH}^{-}$ion (Fig. S3†). So, changing the $\mathrm{pH}$ would be an optional and feasible way to overcome the shortcomings of the chemosensor in sensing fluoride anion from $\mathrm{OH}^{-}$. Specifically, to clarify the effect of the hydroxyl group in the BNB-t4 unit on responses to anions, we replaced the hydroxyl group with a methoxy group to form BNBC-t8. As expected, the selective binding of BNBC-t8 with anions only displayed an obvious variation in absorption spectra upon addition of the same amount of $\mathrm{F}^{-}$and $\mathrm{H}_{2} \mathrm{PO}_{4}{ }^{-}$at room temperature (Fig. 1b). With the addition of 30 equiv. $\mathrm{F}^{-}$ ion, compared to that of BNB-t4, less spectral change for BNBCt8 was observed, indicating a lower sensitivity to $\mathrm{F}^{-}$. The color of the BNBC-t8 in $\mathrm{CHCl}_{3}$ with anions was changed from light yellow to yellow, less color change than that of BNB-t4 and consistent with the absorption spectral observations. The results indicate that the introduction of hydroxy facilitates the binding-induced changes in optical signals, which is in good agreement with previous reports. ${ }^{5,49,50}$ Similar phenomena were also observed for $\mathrm{H}_{2} \mathrm{PO}_{4}{ }^{-}$responsive process (Fig. 1b).

To quantitatively examine the binding properties of BNB-t4 and BNBC-t8 to fluoride anion, we performed the titrated absorption spectral analysis at a low concentration $\left(1 \times 10^{-5} \mathrm{M}\right)$ (Fig. 2 and 3). As shown in Fig. 2, the solution of BNB-t4 in chloroform is light yellow with a dominant absorption maximum at $356 \mathrm{~nm}\left(\pi-\pi^{*}\right.$ transition $)$ and an absorption tail
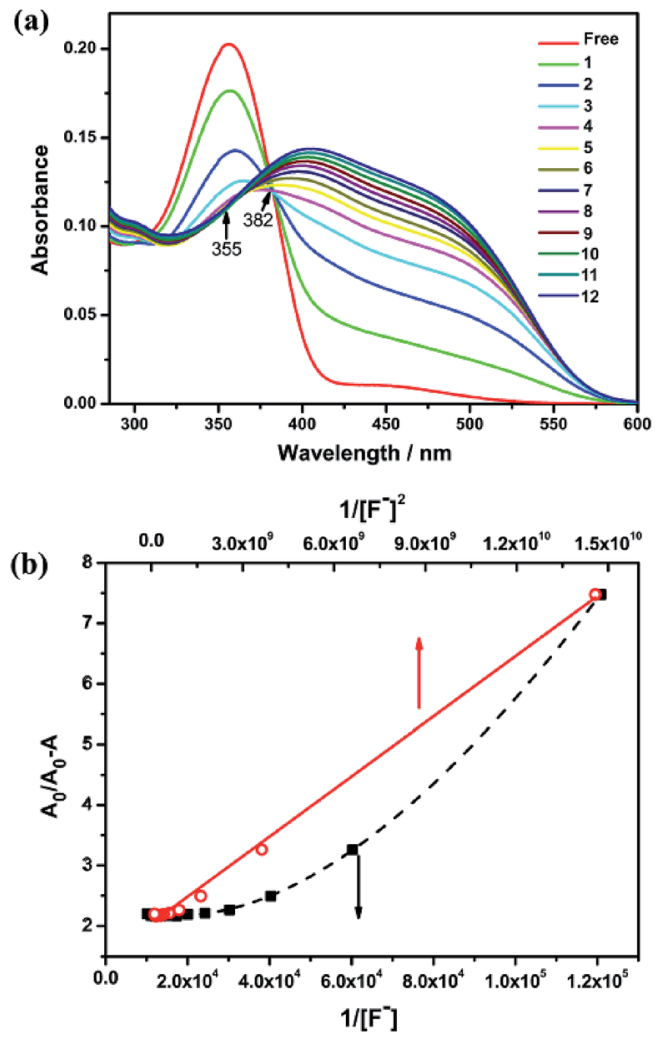

Fig. 2 (a) UV-vis absorption spectra of BNB-t4 $\left(1 \times 10^{-5} \mathrm{M}\right)$ in chloroform titrated with $\mathrm{F}^{-}(0-12$ equiv.) (b) Benesi-Hildebrand plot for complexation of BNB-t4 with $\mathrm{F}^{-}$. $\left[\mathrm{F}^{-}\right]$is the concentration of added $F^{-}(M)$.
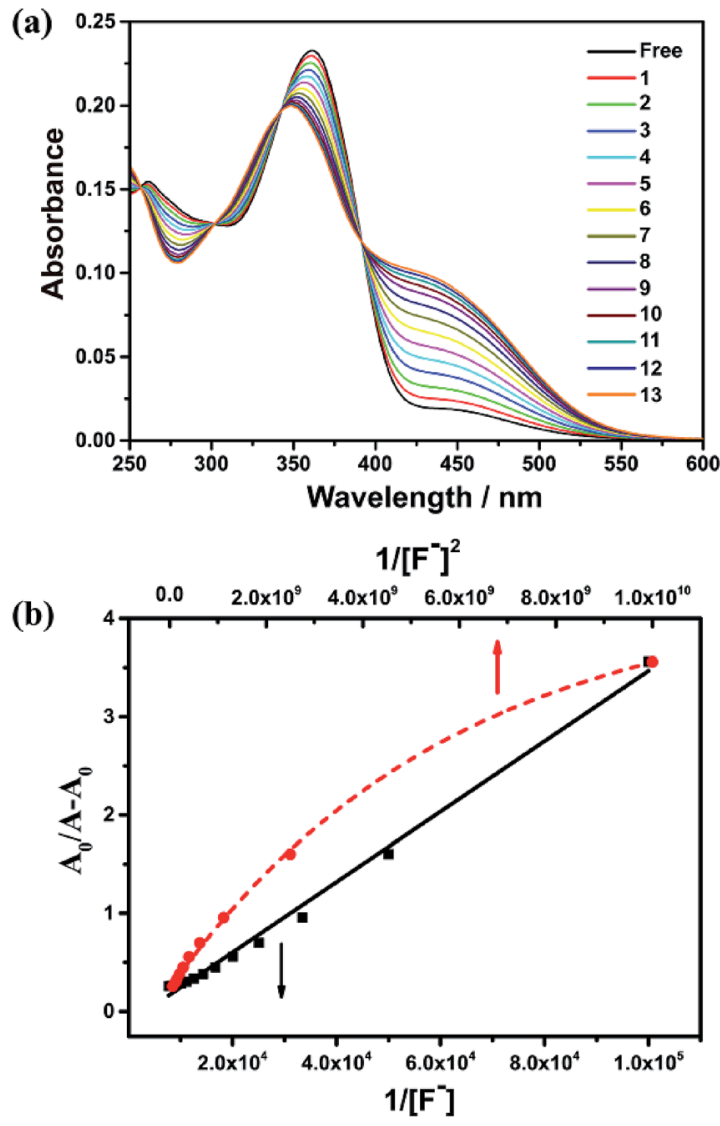

Fig. 3 (a) UV-vis absorption spectra of BNBC-t8 $\left(1 \times 10^{-5} \mathrm{M}\right)$ in chloroform titrated with $\mathrm{F}^{-}(0-13$ equiv.) (b) Benesi-Hildebrand plot for complexation of BNBC-t8 with $\mathrm{F}^{-}$. $\left[\mathrm{F}^{-}\right]$is the concentration of added $\mathrm{F}^{-}(\mathrm{M})$.

around $450 \mathrm{~nm}$. With increasing amount of $\mathrm{F}^{-}$added, the absorbance at $356 \mathrm{~nm}$ gradually decreased and red-shifted to $372 \mathrm{~nm}$ along with a clear isosbestic point at $382 \mathrm{~nm}$, when 4 equiv. of fluoride anions was added. This process corresponds to the formation of a BNB-t4-F $\mathrm{F}^{-}$complex by hydrogen bonding during the first course of titration ( $0-4$ equiv.). With the continuous addition of fluoride anion to 12 equiv., the absorption of $\pi-\pi^{*}$ transition was shifted to $404 \mathrm{~nm}$ and a new isosbestic point was shifted to $355 \mathrm{~nm}$ in the second course. Meanwhile, a new broad absorption band at $460 \mathrm{~nm}$ emerged during the whole titration course, demonstrating the formation of another complex and $\mathrm{HF}_{2}^{-}$by deprotonation of $-\mathrm{NH}$ subgroup. ${ }^{48,59}$ The deprotonation of BNB-t4 upon addition of $\mathrm{F}^{-}$ ion was eventually confirmed by ${ }^{1} \mathrm{H}$ NMR titration as given below (see Fig. 5). Concurrently, the colorimetric changes are striking and visible, from almost colorless to yellow with the addition of fluoride anion. Plotting the $A_{0} /\left(A_{0}-A\right)$ quantity for absorbance at $356 \mathrm{~nm}$ against the reciprocal of $\left[\mathrm{F}^{-}\right]^{2}$ gives a good linear response (Fig. 2b), and shows a $1: 2$ ratio for the complex composition of BNB-t4 and the $\mathrm{F}^{-}$ion in chloroform. ${ }^{59}$ Similarly, this $1: 2$ ratio of complex composition for BNB-t4 with $\mathrm{F}^{-}$ion was also obtained in DMSO (see Fig. S4 $\dagger$ ). Furthermore, the stoichiometric ratio between BNB-t4 and the $\mathrm{F}^{-}$ion was determined by Job's plot and indicated a $1: 2$ 

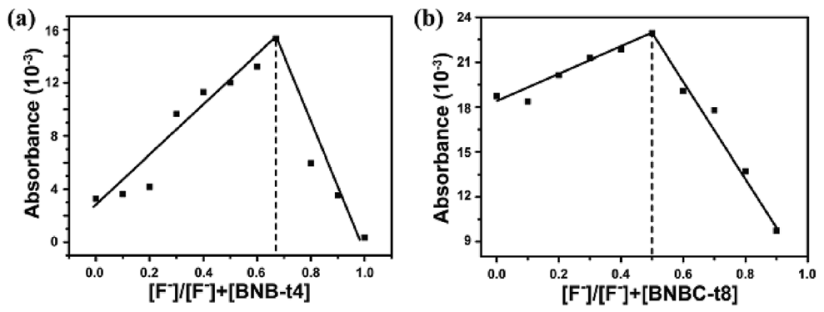

Fig. 4 Job's plots for the complexes of (a) BNB-t4 with $\mathrm{F}^{-}$and (b) BNBC-t8 with $\mathrm{F}^{-}$(in chloroform). Total concentration of [BNB-t4] or $[\mathrm{BNBC}-\mathrm{t} 8]+\left[\mathrm{F}^{-}\right]$was kept constant at $10^{-5} \mathrm{M}$.

binding mode (Fig. 4a), which is consistent with the BenesiHildebrand plot observations. Likewise, two isosbestic points at $302 \mathrm{~nm}$ and $391 \mathrm{~nm}$ were observed in the absorption titration experiments of BNBC-t8 (Fig. 3a). Plotting the $A_{0} /\left(A-A_{0}\right)$ quantity of absorbance at $450 \mathrm{~nm}$ against the reciprocal of $\left[\mathrm{F}^{-}\right]$ (Fig. 3b), by contrast, gives a 1:1 ratio for the complex composition of BNBC-t8 and the $\mathrm{F}^{-}$ion. Moreover, the absorbance value approached the maximum when the molar fraction of BNBC-t8 was 0.5 as shown in Job's plots (Fig. 4b), indicating the formation of a $1: 1$ complex between BNBC-t8 and $\mathrm{F}^{-}$. Taking together, these results suggest that both phenol $\mathrm{O}-\mathrm{H}$ and hydrazide subgroups of BNB-t4 can recognize the added $\mathrm{F}^{-}$ through hydrogen bonding interaction.

Furthermore, the sensitivity and selectivity of BNB-t4 towards anions can also be characterized by the association constant $K_{\mathrm{s}}$ of complex formation between a proton donor and an anion. ${ }^{59}$ As mentioned above, the hydroxyl and hydrazide subgroups in BNB-t4 are the dominant sites for binding fluoride anion, the calculated association constant $K_{\mathrm{s}}$ is as large as 5.54 $\times 10^{9} \mathrm{M}^{-2}$, suggesting efficient and sensitive recognition for $\mathrm{F}^{-}$. In comparison, the association constant of $\mathrm{BNBC}-\mathrm{t} 8$ for $\mathrm{F}^{-}$was determined to be the value of $3.20 \times 10^{3} \mathrm{M}^{-1}$. Thus, the much stronger binding ability of BNB-t4 to $\mathrm{F}^{-}$compared to that of BNBC-t8 can be attributed to $\mathrm{O}-\mathrm{H} / \mathrm{F}$ intermolecular hydrogen bonding. As an important index of chemosensors, the detection limit of receptor BNB-t4 in solution for sensing $\mathrm{F}^{-}$can also be obtained from the plot of absorption as a function of $\mathrm{F}^{-}$ concentration (Fig. S5†), ${ }^{\mathbf{4 1 , 5 9}}$ which was found to be $4.27 \times$ $10^{-8} \mathrm{M}$ in chloroform. In comparison, the detection limit of BNBC-t8 for $\mathrm{F}^{-}$was determined with the value of $2.02 \times 10^{-6} \mathrm{M}$ (Fig. S6 $\dagger$ ), two order smaller than that of BNB-t4. As a consequence, the BNB-t4 with terminal hydroxyl group is more sensitive to fluoride anion than the BNBC-t8 with terminal methoxy group, which can be confirmed by a more visible color change. This high sensitivity for fluoride anion makes the BNBt4 receptor a competitive candidate for environmental detection. In contrast to fluoride detection, the obtained binding ratio is $1: 1$ for BNB-t4 with both $\mathrm{AcO}^{-}$and $\mathrm{H}_{2} \mathrm{PO}_{4}{ }^{-}$anions in chloroform, and the corresponding complex constants are 7.5 $\times 10^{4}\left(\mathrm{M}^{-1}\right)$ and $2.0 \times 10^{3}\left(\mathrm{M}^{-1}\right)$, respectively (Fig. S7 and S8 $\dagger$ ). Therefore, these results indicate that BNB-t4 with terminal hydroxyl groups is more sensitive to fluoride than to other anions.

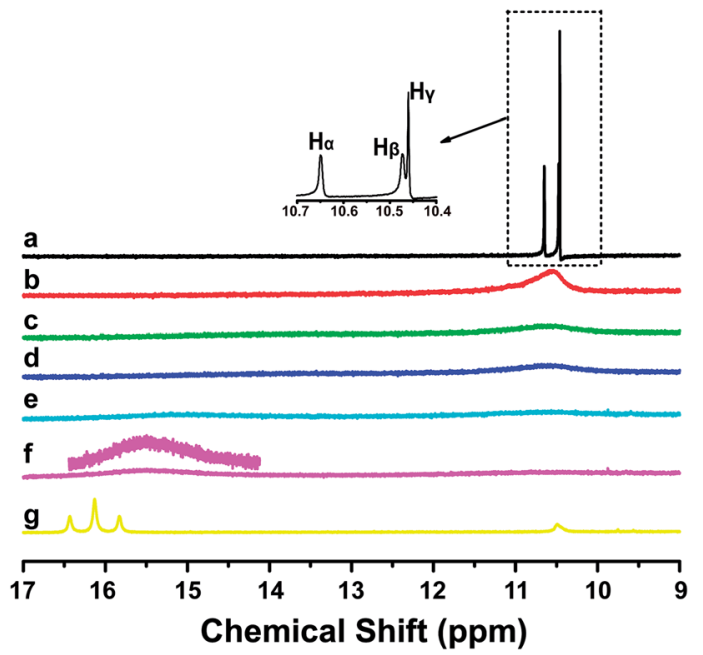

Fig. 5 Partial ${ }^{1} \mathrm{H}$ NMR spectra of BNB-t4 $(5 \mathrm{mM})$ in DMSO- $d 6$ upon the addition of $\mathrm{F}^{-}$(a) free, (b) 0.5 equiv., (c) 2 equiv., (d) 4 equiv., (e) 6 equiv., (f) 8 equiv. and (g) 10 equiv.

To investigate the interaction active sites of BNB-t4 that bind with fluoride anion, the ${ }^{1} \mathrm{H}$ NMR titration experiments were carried out in DMSO- $d_{6}$. Fig. 5 showed the ${ }^{1} \mathrm{H}$ NMR spectra of BNB-t 4 in the titration course of $\mathrm{F}^{-}$and illustrated the spectral shifts of the phenol $\mathrm{O}-\mathrm{H}\left(\mathrm{H}_{\alpha}\right)$ and hydrazide $\mathrm{N}-\mathrm{H}\left(\mathrm{H}_{\beta}\right.$ and $\left.\mathrm{H}_{\gamma}\right)$ group, respectively. The chemical shifts of the $\mathrm{O}-\mathrm{H}$ and $\mathrm{N}-\mathrm{H}$ protons on BNB-t4 appeared at $\delta 10.64,10.47$, and $10.46 \mathrm{ppm}$ before the addition of $\mathrm{F}^{-}$, respectively. After the addition of 0.5 equiv. of $\mathrm{F}^{-}$, the signal of these three protons disappeared, and the simultaneous appearance of a weak broad signal at $\delta 10.56 \mathrm{ppm}$ indicates the formation of $\mathrm{N}-\mathrm{H} \cdots \mathrm{F}^{-}$and $\mathrm{O}-\mathrm{H} \cdots \mathrm{F}^{-}$ hydrogen bonding. The gradual weakening of $\mathrm{H}_{\alpha}, \mathrm{H}_{\beta}$ and $\mathrm{H}_{\gamma}$ proton signals with the increase of $\mathrm{F}^{-}$suggests that the phenol $\mathrm{O}-\mathrm{H}$ and hydrazide $\mathrm{N}-\mathrm{H}$ underwent a deprotonation process. However, after the addition of 10 equiv. fluoride anion, the existence of a weak peak at $10.50 \mathrm{ppm}$ of BNB-t4 indicates that not all of the phenol $\mathrm{O}-\mathrm{H}$ and hydrazide $\mathrm{N}-\mathrm{H}$ experienced a deprotonation process. In the NMR spectra of BNBC-t8 with different amount of $\mathrm{F}^{-}$anion, a weak peak at $10.41 \mathrm{ppm}$ was also observed upon the addition of 6 equiv. $\mathrm{F}^{-}$, suggesting only one of hydrazide $\mathrm{N}-\mathrm{H}$ underwent deprotonation (Fig. S9†). Meanwhile, a new weak triplet signal appeared at $\delta 16.43 \mathrm{ppm}$, 16.13 ppm and 15.83 ppm (Fig. 5g), clearly demonstrates the formation of $\mathrm{HF}_{2}{ }^{-}$. Moreover, the spectral shifts of aromatic phenyl rings $\left(\mathrm{H}_{\delta}, \mathrm{H}_{\varepsilon}\right.$ and $\left.\mathrm{H}_{\omega}\right)$ linked to the hydroxyl and hydrazide group are shown in Fig. S10. $\dagger$ The continuous increase of $\mathrm{F}^{-}$concentration resulted in the protons $\mathrm{H}_{\delta}, \mathrm{H}_{\varepsilon}$ and $\mathrm{H}_{\omega}$ of the phenyl rings to be out of the same chemical environment, and thus the up-field shifts of resonance signals for the $\mathrm{H}_{\delta}, \mathrm{H}_{\varepsilon}$ and $\mathrm{H}_{\omega}$ were observed in the NMR spectra.

Combining all the previous observations and obtained results, the sensing behavior of BNB-t4 for fluoride anions can be schematically described in Scheme $\mathrm{S} 1 . \dagger$ Due to the existence of hydrogen bonding subgroups $-\mathrm{OH}$ and $-\mathrm{NH}$ for fluoride anion in BNB-t4, the addition of $\mathrm{F}^{-}$induces the rebalancing among hydrogen bonding, van der Waals and $\pi-\pi$ stacking in 
solution. Moderate amount of $\mathrm{F}^{-}$ion establishes a hydrogenbonding interaction with BNB-t4 to generate a stable 1:2 complex, while excess amount of fluoride anion causes one of the $\mathrm{NH}$ group to deprotonate, and the other $\mathrm{NH}$ group together with the oxygen atom nearby deprotonated nitrogen atom forms an intramolecular hydrogen bonding. Concomitantly, the recognition of BNB-t4 to fluoride anion leads to colorimetric changes visible to naked eyes in solution. In contrast with the situation of BNBC-t8, the hydrogen bonding subgroup $-\mathrm{NH}$ dominates the recognition of fluoride anion, demonstrating the 1: 1 ratio for the complex composition of BNBC-t8 and $\mathrm{F}^{-}$. Therefore, it can be concluded that the terminal hydroxyl group of BNB-t4 effectively improves the binding ability and the detection limit in sensing fluoride anion, resulting in a more obvious color change that can be sensed by naked eyes. The good performance in binding/sensing fluoride anion over other anions including acetate and phosphate makes BNB-t4 a highly sensitive and selective chemosensor.

To test the ability of receptor BNB-t4 as a colorimetric for fluoride anion against other anions, we carried out competition experiments in the presence of $\mathrm{F}^{-}$mixed with various anions. As shown in Fig. 6, the fluoride anion induced absorption spectral response demonstrates little change with the addition of the other coexistent anions except for $\mathrm{HSO}_{4}{ }^{-}$. The experimental results indicate that the receptor BNB-t 4 has a good selectivity for $\mathrm{F}^{-}$ion in the presence of other anions, making it very useful in practical applications. With the addition of $\mathrm{HSO}_{4}{ }^{-}$, there is no obvious change observed in the absorption spectrum of BNB$\mathrm{t} 4$ without the presence of fluoride anion, indicating a very weak recognition of BNB-t4 to $\mathrm{HSO}_{4}{ }^{-}$(Fig. 6). However, it is very interesting to notice that the absorption spectral change of BNB-t4 induced by $\mathrm{F}^{-}$ion can be efficiently recovered to the initial state with the addition of 10 equiv. $\mathrm{HSO}_{4}{ }^{-}$. In other word, the bonded fluoride anion with BNB-t 4 can be totally released by the addition of $\mathrm{HSO}_{4}{ }^{-}$ion. Correspondingly, the color change of

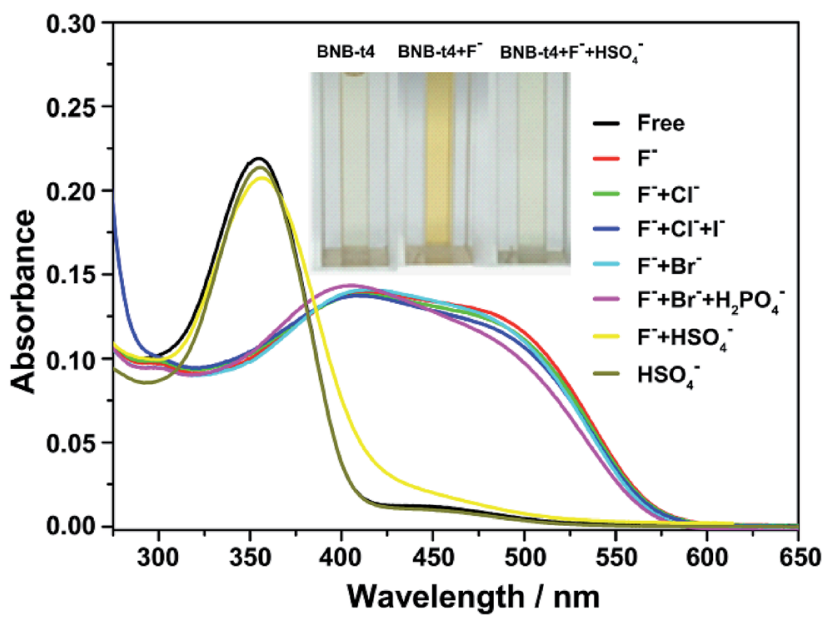

Fig. 6 Competitive selectivity of BNB-t4 $\left(1 \times 10^{-5} \mathrm{~mol} \mathrm{~L}^{-1}\right)$ towards $\mathrm{F}^{-}$ (10 equiv.) in the presence of other anions (10 equiv.) in $\mathrm{CHCl}_{3}$. Inset: the image of BNB-t4 $\left(1 \times 10^{-5} \mathrm{~mol} \mathrm{~L}^{-1}\right)$ in $\mathrm{CHCl}_{3}$ (left), upon addition of 10 equiv. of $\mathrm{F}^{-}$(middle) and BNB-t4 treated with $\mathrm{F}^{-}$(10 equiv.), then added $\mathrm{HSO}_{4}^{-}$(10 equiv.) (right).
BNB-t4 induced by binding $\mathrm{F}^{-}$ion can also be safely switched off with the addition of $\mathrm{HSO}_{4}{ }^{-}$(the inset of Fig. 6), demonstrating a typical OFF-ON-OFF colorimetric sensor with a great recycling feature. Furthermore, the absorption spectral change of BNB-t4 induced by binding $\mathrm{F}^{-}$ion can also be recovered with the addition of $\mathrm{H}^{+}$, such as $\mathrm{HClO}_{4}$ and methanol $(\mathrm{MeOH})$ (Fig. S11†).

The gelation properties of BNB-t4 were discussed in our previous work, and the results indicate that BNB-t4 is capable of forming stable gels in moderately polar solvents such as dichloromethane, chloroform, and aromatic solvents. ${ }^{46}$ The introduction of anions is expected to break the balance among the involved driving forces and induces the phase transition to sense the specific anion in turn. The effect of anions on BNB-t4 gelator was revealed by the gelation experiments in the presence of fluoride anion. When 5 equiv. of $\mathrm{F}^{-}$was added into the chloroform organogel of BNB-t 4 at $25{ }^{\circ} \mathrm{C}$, a thin column of winecolored solution immediately appeared at the upper part and then the gel underwent a gradual decomposition of the gelatinous state in $25 \mathrm{~min}$, yielding a wine-colored solution (Fig. 7). This observation clearly indicates that the force balancing in BNB-t4 gel is destroyed by the introduction of $\mathrm{F}^{-}$and thus causes the dramatic phase transition from gel to sol with the concomitant color change visible to the naked eyes. The changed color of solution can be readily recovered by adding proton reagents such as $\mathrm{MeOH}$. Similar phenomena were also observed upon the addition of solid TBA salts of $\mathrm{AcO}^{-}$or $\mathrm{H}_{2} \mathrm{PO}_{4}{ }^{-}$(Fig. S12 $\dagger$ ), but the corresponding phase transition took a much longer time. This different response rate can be thus used to discriminate fluoride anion from acetate and phosphate anions. However, the color transition and gel decomposition was not observed with the addition of $\mathrm{Cl}^{-}, \mathrm{Br}^{-}$, $\mathrm{I}^{-}$and $\mathrm{HSO}_{4}{ }^{-}$at the identical condition (Fig. S13 $\dagger$ ), indicating the selectivity of BNB-t4 gelator for the recognition of anions. In addition, these results also demonstrate the anions such as $\mathrm{F}^{-}$, $\mathrm{AcO}^{-}$and $\mathrm{H}_{2} \mathrm{PO}_{4}^{-}$, rather than TBA cations, are responsible for the transformation from organogel to solution.

The fluoride anion induced gel-sol transition can be further confirmed by scanning electron microscopy (SEM) study. Without the presence of $\mathrm{F}^{-}$ion, the morphology of BNB-t4 xerogel from chloroform (Fig. 8a) shows entangled and dense fibers. In contrast, these fibers are morphed into bent and swelled sausage structure to expel the gelated solvent (Fig. 8b) with the addition of fluoride anion. Based on the fact that fluoride anion has strong attacking ability to $-\mathrm{NH}$ and $-\mathrm{OH}$

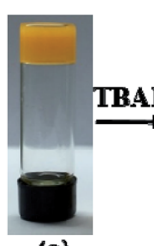

(a)

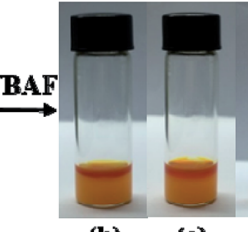

(b) (c)

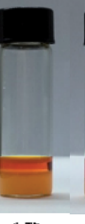

(d)

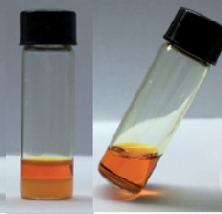

(e)

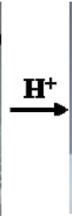

(f)

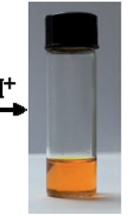

(g)
Fig. 7 (a) Photograph of the chloroform gel of BNB-t4 $\left(8.0 \mathrm{mg} \mathrm{mL}^{-1}\right)$, photographs of the gel after addition of solid TBAF (5 equiv.) in (b) $0.5 \mathrm{~min}$; (c) $3 \mathrm{~min}$; (d) $10 \mathrm{~min}$; (e) $16 \mathrm{~min}$; (f) $25 \mathrm{~min}$, and (g) $20 \mathrm{~min}$ after addition of $0.05 \mathrm{~mL} \mathrm{MeOH}$. 


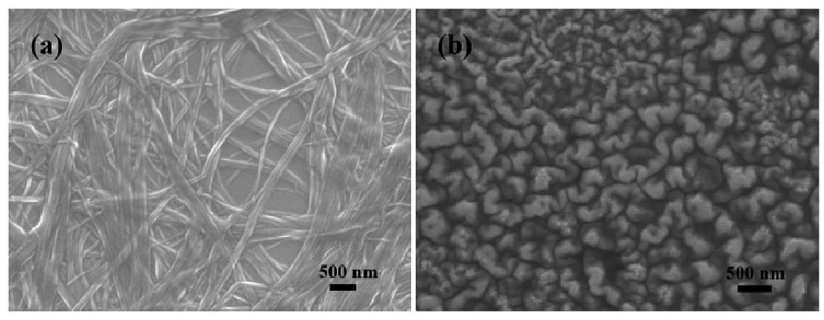

Fig. 8 SEM images of BNB-t4 (a) xerogel from chloroform and (b) chloroform gel treated with $\mathrm{F}^{-}$.

subgroups in BNB-t4, this morphology transformation suggests that $\mathrm{F}^{-}$can be inserted into the molecular assembly by hydrogen binding with the receptor BNB-t4, similar to the binding mode in solution. As a result, the host-guest binding destroys the balance of intermolecular interactions and initiates the driving force competition with the introduction of $\mathrm{F}^{-}$. It is however worthwhile to mention that this phase transition could also be partly attributed to the deprotonation of - $\mathrm{NH}$ and -OH subgroups, especially in the case of excess $\mathrm{F}^{-}$. As a consequence of the deprotonation, a new established balance among the driving forces consisting of hydrogen bonding, van der Waals and $\pi-\pi$ stacking results in the formation of final solution phase.

\section{Experimental}

\section{Materials}

All the solvents for spectral measurements were of spectroscopic grade and used as received. Tetrabutylammonium salts were all $>98 \%$ pure and dried in vacuum overnight before use. The solutions used in titrations were prepared from freshly opened solvent bottles.

\section{Synthesis and characterization}

The synthesis and characterization of receptors BNB-t 4 and BNBC-t8 were described in our previous work. ${ }^{46,58}$ The UV-vis absorption spectra were obtained on a Cary 5000 UV-vis-NIR spectrometer with quartz cuvettes of the appropriate path length $(0.1-1 \mathrm{~cm})$ at room temperature. ${ }^{1} \mathrm{H}$ NMR spectra were recorded with an Avance- $400400 \mathrm{MHz}$ spectrometer using tetramethylsilane (TMS) as an internal standard. Field emission scanning electron microscopy (FE-SEM) images were taken with a JSM-6700F apparatus. Samples for FE-SEM measurement were prepared by wiping a small amount of gel or solution onto a silicon plate and followed by drying in a vacuum for $12 \mathrm{~h}$ at room temperature.

Spectrophotometric titrations. Titrations were performed at room temperature in chloroform. In a typical experiment, the solution of the receptor (BNB-t4 and BNBC-t8) was titrated with a 200-fold concentrated solution of the tetrabutylammonium salt of the desired anion. The $\mathrm{pH}$ dependence experiment was performed in HEPES buffer solution system (DMSO : $\mathrm{H}_{2} \mathrm{O}=$ $80: 20[\mathrm{v} / \mathrm{v}]$, containing $0.01 \mathrm{~mol} \mathrm{~L}^{-1}$ HEPES, $\left.\mathrm{pH}=1-14\right)$.

Gelation test. Weighted gelator was mixed in a cap sealed test tube $[3.5 \mathrm{~cm}$ (height) $\times 0.5 \mathrm{~cm}$ (radius) $]$ with an appropriate amount of solvent, and the mixture was heated until the solid dissolved. The sample vial was cooled to $4{ }^{\circ} \mathrm{C}$ and then turned upside down. When a clear or slightly opaque gel formed, the solvent therein was immobilized at this stage.

\section{Conclusions}

The dually responsive properties of BNB-t4 bearing hydrazide and azobenzene groups in the recognition of fluoride anion have been systematically studied in this work. The selective response to fluoride anion over other anions indicates that the BNB-t4 is a good candidate in recognizing fluoride anion. The interactions of fluoride anion with amidic - $\mathrm{NH}$ and hydroxyl proton of BNB-t4 via hydrogen bonding and deprotonation cause the formation of new complexes and regulate their electronic states and molecular configurations. As a consequence, the remarkable changes in the absorption of receptor BNB-t4 with terminal hydroxyl group demonstrate a colorimetric chemosensor with better selectivity and higher sensitivity in sensing fluoride anion than BNBC-t8 with terminal methoxy group. It can be concluded that the introduction of hydroxyl group greatly improves the recognition ability and detection limit of BNB-t4 for sensing fluoride anion in solution. Meanwhile, the anion induced gel-sol transition of BNB-t4 selfassembly demonstrates a potential application in sensing fluoride anion through the noticeable color change and phase transition. Meanwhile, the proposed sensing mechanism and binding ratio for BNB-t4 with fluoride anion has been used to interpret the observations both in solution phase and in gel phase. Moreover, the color change of BNB-t4 induced by binding fluoride anion can be safely switched off with the addition of $\mathrm{HSO}_{4}{ }^{-}$, indicating that the BNB-t4 is a typical OFF$\mathrm{ON}-\mathrm{OFF}$ colorimetric sensor with a great reversible feature. Therefore, the dual responsive receptor BNB-t4 with high selectivity and sensitivity for fluoride anion could be a competitive chemosensor candidate for environmental applications.

\section{Conflicts of interest}

There are no conflicts to declare.

\section{Acknowledgements}

The authors are grateful to the National Natural Science Foundation of China (projects No. U1504510, U1604129, U1404619 and 21173068).

\section{Notes and references}

1 N. Busschaert, C. Caltagirone, W. V. Rossom and P. A. Gale, Chem. Rev., 2015, 115, 8038.

2 E. Gazzano, L. Bergandi, C. Riganti, E. Aldieri, S. Doublier, C. Costamagna, A. Bosia and D. Ghigo, Curr. Med. Chem., 2010, 17, 2431.

3 J. L. Baker, N. Sudarsan, Z. Weinberg, A. Roth, R. B. Stockbridge and R. R. Breaker, Science, 2012, 335, 233. 4 V. Bhalla, H. Singh and M. Kumar, Org. Lett., 2010, 12, 628. 
5 Y. Zhou, J. F. Zhang and J. Yoon, Chem. Rev., 2014, 114, 5511. 6 E. Newbrun, J. Public Health Dent., 2010, 70, 227.

7 H. S. Horowitz, J. Public Health Dent., 2003, 63, 3.

8 N. Kumari, S. Jha and S. Bhattacharya, J. Org. Chem., 2011, 76, 8215.

9 X.-L. Liu, M. Mao, M.-G. Ren, Y. Tong and Q.-H. Song, Sens. Actuators, B, 2014, 200, 317.

10 Madhuprasad, A. N. Shetty and D. R. Trivedi, $R S C A d v ., 2012$, 2, 10499.

11 W. Tan, D. Zhang and D. Zhu, Bioorg. Med. Chem. Lett., 2007, 17, 2629.

12 S. Madhu and M. Ravikanth, Inorg. Chem., 2014, 53, 1646.

13 J.-S. Chen, P.-W. Zhou, G.-Y. Li, T.-S. Chu and G.-Z. He, J. Phys. Chem. B, 2013, 117, 5212.

14 H. Zhang, J. Hu and D.-H. Qu, Org. Lett., 2012, 14, 2334.

15 M. E. El-Khouly, A. N. Amin, M. E. Zandler, S. Fukuzumi and F. D'Souza, Chem.-Eur. J., 2012, 18, 5239.

16 O. A. Bozdemir, F. Sozmen, O. Buyukcakir, R. Guliyev, Y. Cakmak and E. U. Akkaya, Org. Lett., 2010, 12, 1400.

17 Y. Sun, N. Ross, S.-B. Zhao, K. Huszarik, W.-L. Jia, R.-Y. Wang, D. Macartney and S. Wang, J. Am. Chem. Soc., 2007, 129, 7510.

18 J. Wang, F.-Q. Bai, B.-H. Xia, L. Sun and H.-X. Zhang, J. Phys. Chem. A, 2011, 115, 1985.

19 R. B. P. Elmes and T. Gunnlaugsson, Tetrahedron Lett., 2010, 51, 4082.

20 C. R. Wade, A. E. J. Broomsgrove, S. Aldridge and F. P. Gabbai, Chem. Rev., 2010, 110, 3958.

21 W. Xu, S. Liu, H. Sun, X. Zhao, Q. Zhao, S. Sun, S. Cheng, T. Ma, L. Zhou and W. Huang, J. Mater. Chem., 2011, 21, 7572 .

22 M. Xue, X. Wang, L. Duan, W. Gao, L. Ji and B. Tang, Biosens. Bioelectron., 2012, 36, 168.

23 J. Hu, G. Zhang, Y. Geng and S. Liu, Macromolecules, 2011, 44, 8207.

24 M. Xue, X. Wang, H. Wang, D. Chen and B. Tang, Chem. Commun., 2011, 47, 4986.

25 T.-H. Kim and T. M. Swager, Angew. Chem., Int. Ed., 2003, 42, 4803.

26 S. Goswami, A. K. Das, A. Manna, A. K. Maity, H.-K. Fun, C. K. Quah and P. Saha, Tetrahedron Lett., 2014, 55, 2633.

27 C. Saravanan, S. Easwaramoorthi, C.-Y. Hsiow, K. Wang, M. Hayashi and L. Wang, Org. Lett., 2014, 16, 354.

28 J.-S. Chen, P.-W. Zhou, L. Zhao and T.-S. Chu, RSC Adv., 2014, 4, 254.

29 K. Kanagaraj and K. Pitchumani, Chem.-Asian J., 2014, 9, 146. 30 J.-S. Chen, P.-W. Zhou, S.-Q. Yang, A.-P. Fu and T.-S. Chu, Phys. Chem. Chem. Phys., 2013, 15, 16183.

31 F. Wang, J. Wu, X. Zhuang, W. Zhang, W. Liu, P. Wang and S. Wu, Sens. Actuators, B, 2010, 146, 260.

32 Q. Song, A. Bamesberger, L. Yang, H. Houtwed and H. Cao, Analyst, 2014, 139, 3588.

33 L. Gai, H. Chen, B. Zou, H. Lu, G. Lai, Z. Li and Z. Shen, Chem. Commun., 2012, 48, 10721.

34 M. Cametti and K. Rissanen, Chem. Soc. Rev., 2013, 42, 2016. 35 V. Amendola, G. Bergamaschi, M. Boiocchi, L. Fabbrizzi and L. Mosca, J. Am. Chem. Soc., 2013, 135, 6345.
36 M. Wenzel, J. R. Hiscock and P. A. Gale, Chem. Soc. Rev., 2012, 41, 480.

37 Q.-Y. Cao, T. Pradhan, M. H. Lee, K. No and J. S. Kim, Analyst, 2012, 137, 4454.

38 (a) B. Bai, J. Ma, J. Wei, J. Song, H. Wang and M. Li, Org. Biomol. Chem., 2014, 12, 3478; (b) X. Gu, B. Bai, H. Wang and M. Li, $R S C A d v ., 2017,7,218$; (c) J. Wei, Q. Chai, L. H. He, B. L. Bai, H. T. Wang and M. Li, Tetrahedron, 2016, 72, 3073; (d) B. Bai, X. Mao, J. Wei, Z. Wei, H. Wang and M. Li, Sens. Actuators, B, 2015, 211, 268; (e) S. Ghosh, A. Ganguly, A. Bhattacharyya, M. A. Alam and N. Guchhait, RSC Adv., 2016, 6, 67693.

39 D. H. Lee, K. H. Lee and J.-I. Hong, Org. Lett., 2001, 3, 5.

40 Y. M. Hijji, B. Bararea, A. P. Kennedy and R. Butcher, Sens. Actuators, B, 2009, 136, 297.

41 X. Zhang, J. Fu, T.-G. Zhan, L. Dai, Y. Chen and X. Zhao, Tetrahedron Lett., 2013, 54, 5039.

42 Q. Li, Y. Guo, J. Xu and S. Shao, Sens. Actuators, B, 2011, 158, 427. 43 R. Dutzler, E. B. Campbell, M. Cadene, B. T. Chait and R. MacKinnon, Nature, 2002, 415, 287.

44 G. Yu, X. Yan, C. Han and F. Huang, Chem. Soc. Rev., 2013, 42, 6697.

45 A. Pal, S. Shrivastava and J. Dey, Chem. Commun., 2009, 6997. 46 Y. Li, X. Ran, Q. Li, Q. Gao and L. Guo, Chem.-Asian J., 2016, 11, 2157.

47 T. H. Kim, M. S. Choi, B. Sohn, S. Park, W. S. Lyoo and T. S. Lee, Chem. Commun., 2008, 20, 2364.

48 L. Xing, B. Yang, X. Wang, J. Wang, B. Chen, Q. Wu, H. Peng, L. Zhang, C. Tung and L. Wu, Langmuir, 2013, 29, 2843.

49 L. Zang, H. Shang, D. Wei and S. Jiang, Sens. Actuators, B, 2013, 185, 389.

50 Y. Zhang, Q. Lin, T. Wei, X. Qin and Y. Li, Chem. Commun., 2009, 40, 6074.

51 P. Xue, Y. Zhang, J. Jia, D. Xu, X. Zhang, X. Liu, H. Zhou, P. Zhang, R. Lu, M. Takafujic and H. Iharac, Soft Matter, 2011, 7, 8296.

52 Q. Lin, X. Zhu, Y. Fu, Y. Zhang, R. Fang, L. Yang and T. Wei, Soft Matter, 2014, 10, 5715.

53 Z. Džolić, M. Cametti, A. D. Cort, L. Mandolini and M. Žinić, Chem. Commun., 2007, 34, 3535.

54 J. Liu, J. Ma and C. Chen, Tetrahedron, 2011, 67, 85.

55 J. Liu, Y. Yang, C. Chen and J. Ma, Langmuir, 2010, 26, 9040. 56 (a) Q. Lin, T. Lu, X. Zhu, B. Sun, Q. Yang, T. Wei and Y. Zhang, Chem. Commun., 2015, 51, 1635; (b) Q. Lin, K. Zhong, J. Zhu, L. Ding, J. Su, H. Yao, T. Wei and Y. Zhang, Macromolecules, 2017, 50, 7863; (c) Q. Lin, P. Mao, Y. Fan, P. Jia, J. Liu, Y. Zhang, H. Yao and T. Wei, Soft Matter, 2017, 13, 7360; (d) Q. Lin, P. Mao, Y. Fan, L. Liu, J. Liu, Y. Zhang, H. Yao and T. Wei, Soft Matter, 2017, 13, 7085; (e) Q. Lin, T. Lu, X. Zhu, T. Wei, H. Li and Y. Zhang, Chem. Sci., 2016, 7, 5341.

57 C. Deng, R. Fang, Y. Guan, J. Jiang, C. Lin and L. Wang, Chem. Commun., 2012, 48, 7973.

58 X. Ran, H. Wang, P. Zhang, B. Bai, C. Zhao, Q. Zhang, H. Liu, Z. Yu and M. Li, Liq. Cryst., 2011, 38, 1227.

59 Q. Chu, D. A. Medvetz and Y. Pang, Chem. Mater., 2007, 19, 6421. 